\title{
Probabilidade de morrer no primeiro ano de vida em área urbana da região sul, Brasil ${ }^{*}$
}

\author{
Probability of dying in the first year of life in an urban area of Brazil
}

\author{
Regina K. Tanno de Souza**, Sabina L.D. Gotlieb***
}

\begin{abstract}
SOUZA, R.K.T. de \& GOTLIEB, S.L.D. Probabilidade de morrer no primeiro ano de vida em área urbana da região sul, Brasil. Rev.Saúde Pública, 27: 445-54, 1993. Coorte constituída por 4.876 crianças nascidas vivas, em hospitais do Município de Maringá-PR (Brasil), em 1989, foi acompanhada com a finalidade de estimar a probabilidade de morrer no primeiro ano de vida. As variáveis de estudo foram sexo, peso ao nascer, idade da mãe, subgrupos etários e causa básica de morte. A probabilidade de morte no primeiro ano de vida foi estimada em 19,9 por mil, sendo que $77,3 \%$ dos óbitos ocorreram no período neonatal. As causas perinatais, juntamente com as anomalias congênitas, responderam por mais de $80 \%$ dos óbitos, e as doenças infecciosas e parasitárias, por apenas $1,1 \%$. A probabilidade de morrer no primeiro ano de vida devido às afecçðes originadas no período perinatal foi superior nas crianças nascidas de parto normal (20,3 por mil) em relação à das nascidas por cesárea ( 9 por mil). O risco de morrer foi maior nos filhos de mulheres adolescentes, nas crianças nascidas com peso inferior a $2.500 \mathrm{~g}$. Os resultados chamam a atenção para a necessidade de melhorar a qualidade da assistência pré-natal, ao parto e ao recém-nascido e sugerem possível associação entre maior mortalidade e pior nível socioeconômico.
\end{abstract}

Descritores: Mortalidade infantil. Probabilidade. Fatores de risco.

\section{Introdução}

Inúmeros trabalhos têm abordado a mortalidade infantil, analisando-a quanto a sua magnitude. Sua relevância é consenso no mcio científico, cuja importância não se restringe a este meio mas se estende aos contextos econômico, político e social.

$O$ cocficiente de mortalidade infantil-CMI(relação cntre os númcros de óbitos de menores de um ano e de nascidos vivos) ${ }^{9}$ tcm sido reconhecido como um dos indicadores mais sensiveis da condição de saúde ${ }^{3,12,13}$, bastante utilizado para comparação entre diferentes regiōcs ${ }^{8}$. É medida que reflete as condições de vida de uma população e se

* Financiado pela FAPESP, Processo 91/3069-0. Parte da Dissertação apresentada ao Departamento de Epidemiologia da Faculdade de Saúde Pública da Universidade de São Paulo, em 1992.

* Departamento de Enfermagem da Fundação Universidade Estadual de Maringá - Maringá, PR - Brasil

*** Departamento de Epidemiologia da Faculdade de Saúde Pública da Universidade de São Paulo - São Paulo, SP Brasil

Separatas/Reprints: R.K.T.de Souza - Av. Colombo, 3690 87020-900 - Maringá, PR - Brasil

Edição subvencionada pela FAPESP. Processo Medicina 93/ 0208-5. apóia na influência que os principais determinantes do nível de vida como alimentação, moradia, acesso ao conhecimento médico e seu desenvolvimento, entre outros, exercem sobre a probabilidade de sobreviver ao primeiro ano de vida.

Conforme Simões ${ }^{18}$, a mortalidade infantil vem aprescntando tendência declinante, em nível mundial, fenômeno que vem se processando de forma desigual e com determinantes específicos, de acordo com o nivel de desenvolvimento de cada área. Assim, $\mathrm{cm}$ países industrializados, o progresso socioeconômico, sem dúvida, desempenhou importante papel e, nas sociedades em vias de desenvolvimento, as causas da diminuição parecem ter sido outras ${ }^{1}$. Os países não participantes da revolução ocorrida no século passado registraram reduçőes tardias em seu nível de mortalidade ${ }^{13}$ que são creditadas principalmente a intervenções setoriais, nem scmpre acompanhadas de desenvolvimento socioeconômico integral ${ }^{1}$. Vúrios autores ${ }^{5,13,18}$ atribuem a rcdução da mortalidade infantil, nas últimas décadas, no Brasil, ao saneamento básico e, mais recentemente, aos avanços específicos da medicina e da saúde pública. Alćm desses fatores, assinalam-se modificações nos padrões da fecundidade e da natalidade, nos últimos anos, como responsávcis pela alıcração da cvolução da mortalidade infantil ${ }^{12}$.

$\mathrm{Na}$ atualidade encontram-se, de um lado, paises que aprescntam estimativas próximas a 15 por mil 
nascidos vivos (n.v.) e, por outro, regiōes classificadas como menos desenvolvidas (África e América-Latina), com valores que supcram 80 por mil n.v. ${ }^{10}$. Esses diferenciais observados e a multiplicidade de situações que determinam maior ou menor risco de morte apontam a importância de abordagens que se voltem, simultaneamente, para o estabclecimento de associações com os aspectos globais, bcm como com os de natureza específica.

Tradicionalmente, os cstudos sobre a mortalidade infantil partem dos dados já existentes, geralmente com basc nas cstatísticas vitais oficiais, com cálculo de cocficientes. Esses estudos aprescntam algumas limitaçõcs:

- as estatísticas oficiais podem apresentar distorções $\mathrm{c}$ subestimar o número de nascimentos c/ou 6bitos, não revclando a real magnitude do problema;

- embora o coeficiente seja entendido como cstimativa de risco, possui vićs que pode assumir imporlância se ocorrercm variaçōes bruscas no número de nascimentos de um ano a outro ${ }^{5}$;

- no cstudo de fatores associados, devido a insuficiência de informaçõcs, pois as cstatísticas oficiais são apresentadas somente segundo scxo, idade c causa básica, cnquanto que outras variáveis fortemente associadas à mortalidade infantil não são disponívcis, como peso ao nascer $\mathrm{c}$ idade da mãc.

Frentc às questõcs colocadas, o presente estudo constitui uma tentativa de aprofundar o conhecimento sobre a mortalidade infantil $\mathrm{cm}$ Maringá $\Gamma R$, onde o coeficiente de mortalidade infantil reduziu-sc $\mathrm{cm} 41,6 \%$ no período 1980/88, atingindo patamares próximos a 20 por mil n .v., diferindo de várias localidades brasilciras $\mathrm{c}$ conferindo uma cspecificidade, principalmente, naquilo que se refere à adoção de medidas que viscm a reduções futuras.

O objetivo da presente pesquisa é estimar a probabilidade de morte infantil de coorte constituida por crianças nascidas $\mathrm{cm}$ hospitais do Município de Maringá - PR, filhos de mulheres ncle rcsidentes, cm 1989, segundo subgrupos ctários, causas básicas, peso ao nascer, sexo c idade da mãc.

\section{Metodologia}

\section{População de estudo e fontes de dados}

Constituíram a população de cstudo 4.876 nascidos vivos, cujos partos realizaram-sc nos hospitais do Município de Maringá, entre 1 de janciro c 31 de dezembro de 1989, filhos de mulheres residentes.
A coleta foi realizada nos sete hospitais do Municipio que prestam atendimento ao parto, e no Centro de Epidemiologia da Secretaria Estadual de Saúde do Paraná. Foram consultados os livros de registro de nascimentos existentes em scis hospitais (um hospital não faz anotação em livro), os livros de registro de todas internações, - livro da maternidade e o prontuário médico (ncsta ordem de utilização) para complementar informações essenciais.

Os dados referentes aos óbitos foram levantados das primeiras vias das declaraçōes de óbito (DO) arquivadas na Secretaria de Saúde do Paraná (SSP).

\section{Procedimentos}

O levantamento dos dados foi realizado diretamente em planilhas $\mathrm{c}$, no ato da coleta, os dados cram codificados conforme padronização prévia.

Foram cxcluídos da coleta, os nascimentos que continham a obscrvação "nascido morto", "feto morto" ou "natimorto", e os que fizessem menção à residência $\mathrm{cm}$ outros municípios.

Quando ocorria um óbito infantil, no pcríodo intra-hospitalar, já na colcta era devidamente destacado.

Concluída a colcta da coorte de nascimentos, foi realizado o levantamento dos óbitos, através das primeiras vias das DO arquivadas no Centro de Epidemiologia da SSP. Inicialmente, foram coletadas todas as DO das crianças nascidas em 1989 , naturais de Maringá, que faleceram no primciro ano de vida. Fizeram também parte desta seleção as declarações de nascidos mortos, em função do possível mau preenchimento da DO, isto $c$, um nascido vivo que viesse a falccer imediatamentc após o parto $\mathrm{c}$ fosse declarado como nascido morto?

Foram localizadas 103 DO dc crianças naturais do Município de Maringá, cujo cvento ocorreu no Estado do Paraná; posteriormentc, algumas foram excluidas $\mathrm{c}$ outras acrescentadas.

De posse das DO, tentou-se identificar aquelas que se referiam à coorte definida. Foram utilizadas, inicialmente, a listagem que relaciona os nascidos vivos registrados, pois dado que a criança nasceu viva c foi a óbito, o espcrado ć que tenham sido realizados dois registros: o de nascimento c o de óbito. Nessa listagem, das 103 dcclaraçōes sclecionadas, 86 foram localizadas cntre os registros de nascimento (Figura). As demais (17) foram avaliadas individualmente quanto à sua inclusão, vcrificando-se que 5 refcriamsc à população estudada, totalizando até aqui 91 óbitos. Alćm destes, mais scis óbitos foram acrescentados: 


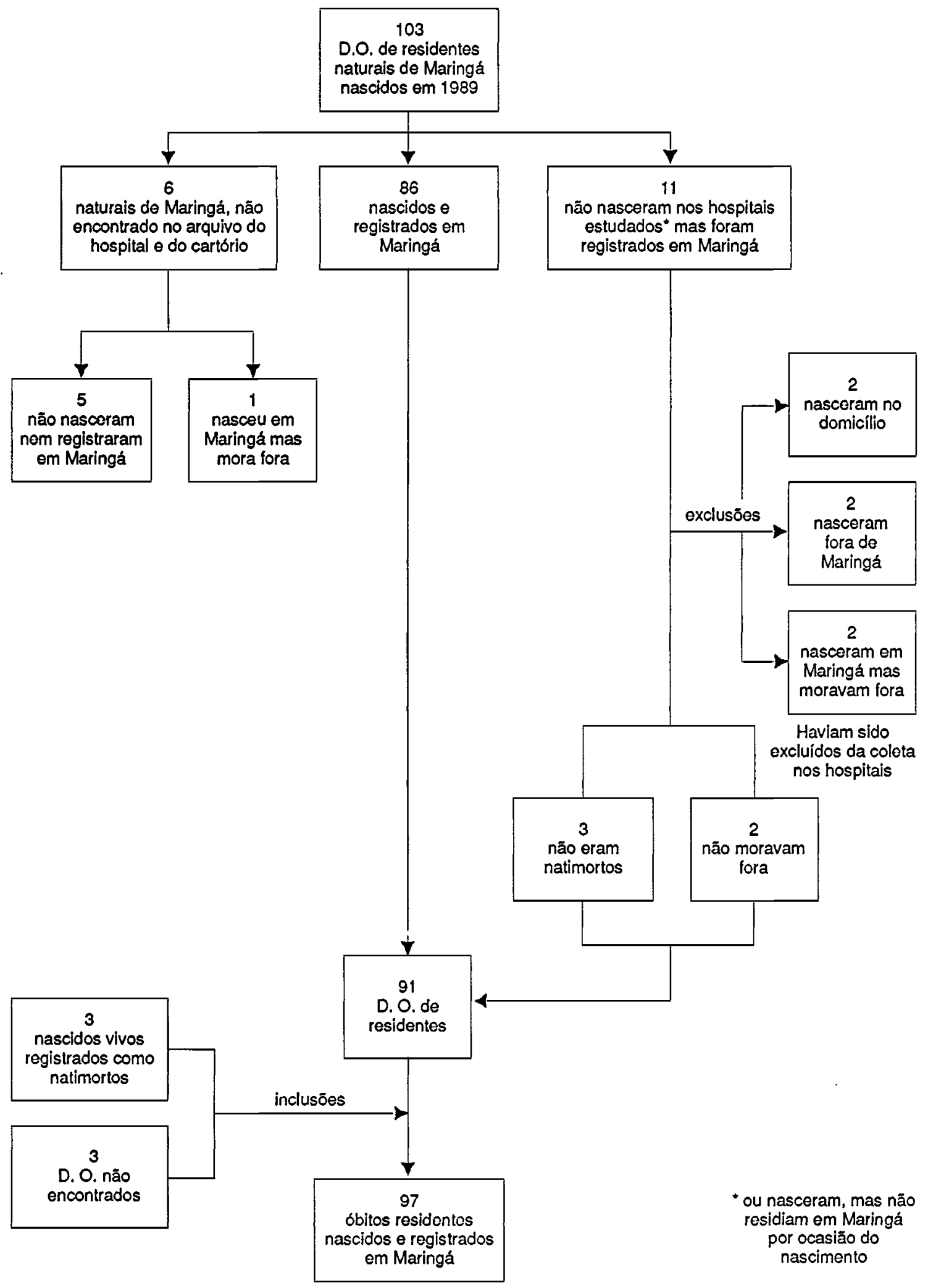

Figura. Fluxo da seleção da população de óbitos. 
- Lrês cram crianças registradas como nascidas mortas, mas, nas anotaçōcs hospitalares, constava que estas crianças nasccram com vida e faleccram logo após o nascimento. Reafirma esta informação a própria $\mathrm{DO}$, na qual consta no campo referente a "Morte (cm rclação ao parto)", que a criança foi a óbito após o parto;

- três eram crianças falecidas no período intrahospitalar, cujas declaraçðes não foram localizadas no Centro de Epidcmiologia. Duas eram crianças devidamente registradas quanto ao nascimento e óbito; a outra corresponde a uma situação na qual ocorreu simultaneamente o subregistro de nascimento e o de óbito. As evidências não deixam dúvidas que a criança nasceu com vida, pois o prontuário médico faz referência a retirada de um concepto com vida, apresentando anomalias e hidrocefalia. Estes três úllimos casos correspondem àqucles cuja causa de morte é ignorada. As demais variávcis foram analisadas a partir dos dados dos hospitais.

Desta forma, 97 crianças nascidas $\mathrm{cm}$ hospitais de Maringá, cm 1989, filhos de mulheres residentes foram a óbito antes de completar um ano de vida (Figura).

\section{Delineamento da pesquisa e medidas utilizadas}

A pesquisa é do tipo longitudinal em que a coortc foi formada por 4.876 crianças nascidas vivas $\mathrm{cm}$ hospital $\mathrm{c}$ foram rastreadas até completar um ano de idade, por meio das DO respectivas, com a finalidade de medir a ocorrência do óbito.

Probabilidade de morte infantil ${ }^{6}$ - Foi obtida relacionando-se os óbitos de menores de um ano de uma geração de nascimentos hospitalares com estes nascimentos. É uma cstimativa da probabilidade minima, pois óbitos ocorridos fora do Estado do Paraná c casos $\mathrm{cm}$ que não foram prcenchidas as DO, são siluaçōes que podem ter ocorrido, mas não foram de alcance do presente csludo.
- Subgrupos etários: óbito neonatal precoce (menores de 7 dias), neonatal tardio (de 7 a 27 dias) e pós-neonatal (28 dias e mais).

- Causa básica de morte: classificados nos 17 Capítulos de causas e em algumas categorias da Classificação Internacional de Doenças - 9ª Revisão ${ }^{11}$.

- Idade materna: expressa em anos, apresentando-se em grupos qüinqüenais.

\section{Resultados e discussão}

Das 4.876 crianças nascidas vivas, 97 faleceram antes de completar um ano de vida. Portanto, a estimativa da probabilidade de morrer entre menores de um ano foi igual a 19, 9 por mil, valor este semelhante ao do Coeficiente de Mortalidade Infantil, obtido através dos dados oficiais $(22,6$ por mil n.v., para o mesmo ano). A pequena diferença entre as duas medidas de mortalidade pode ser atribuída a:

1. no estudo foram excluídas as crianças nascidas $\mathrm{cm}$ domicílio. No registro oficial, foi verificado que, $\mathrm{cm}$ 1989, 20 crianças nasceram em domicílio e destas, 2 foram a óbito. A inclusão dessas crianças faria com que a probabilidade passasse de 19,9 para 20,2 por mil.

2. diferença existente entre os cálculos da probabilidade e do coeficiente.

3. possível subestimativa do denominador no cálculo de coeficientes que, via de regra, é represcntado pelas crianças registradas $\mathrm{em}$ cartório, o que nem sempre corresponde à totalidade de nascimentos de uma dada árca. Em trabalho anterior ${ }^{20}$ a estimativa do subregistro de nascimento nessa população foi igual a $9,1 \%$.

A mortalidade infantil cm Maringá, cm 1989, pode ser considerada relativamente baixa, mesmo

$$
\underset{\text { Pe }}{\begin{array}{c}
\text { Probalidade } \\
\text { morte infantil }
\end{array}}=\frac{\text { Óbitos de crianças menores de } 1 \text { ano nascidas em hospitais de Maringá (residentes) em } 1989}{\text { Total de nascidos vivos em hospitais de Maringá (residentes) em } 1989} \times 1000
$$

\section{Variáveis de estudo}

As variávcis consideradas $\mathrm{c}$ respectivas modalidades foram:

\section{Sexo: masculino e fominino.}

- Peso: cxpresso cm gramas c considerando as calegorias: baixo peso ao nascer (peso inferior a $2.500 \mathrm{~g})$, peso inadequado ao nascer $(2.500$ a $2.999 \mathrm{~g}$ ) e peso normal ao nascer (igual ou superior a $3.000 \mathrm{~g}$ ). quando comparada à estimativa para o país, como um todo, cm 1987 (53 por mil n.v.) e de algumas regiōcs brasilciras, para csse mesmo ano, como Sul $(37,0$ por mil n.v.), Sudeste $(38,4$ por mil n.v.) c Nordesic $(74,7 \text { por mil n.v. })^{19}$. Por outro lado, a taxa ć alıa, se comparada a de países desenvolvidos. A projeção da mortalidade para o periodo 1950 - 2025, realizada pelas Nações Unidas ${ }^{10}$, estima para o periodo atual $(1985-1990)$ uma mortalidade infantil da ordem de $9,9,7,2 \mathrm{c}$ 6,5 por mil n.v. para Canadá, Japão e Suécia, res- 
pectivamente. Ainda considerando esta projeção, observa-se que o risco de morrer das crianças maringaenses está bem distante ao da América Latina (55,7 por mil n.v.) e mais se aproxima ao de Cuba (18,6 por mil n.v.).

Contudo, Maringá, a exemplo de alguns outros municípios brasileiros, como São Caetano do Sul no Estado de São Paulo (CMI = 18,8 por mil n.v.) ${ }^{17}$ apresenta taxa diferenciada frente à realidade nacional. Conforme exaustivamente explorada pela literatura, a provável explicação encontra-se no campo econômico. O Município conta com uma agropecuária de expressão, o comércio e a indústria são capazes de absorver inclusive parcela de trabalhadores de municípios circunvizinhos. Certamente este potencial econômico contribui de forma favorável à geração do que Kowarick ${ }^{7}$ denomina salários indiretos, isto é, "os gastos realizados em educação, saúde (...), moradia (...), elementos necessarios à vida coletiva...", que são conseqüentes às possibilidades de captação de recursos a serem geridos pelo setor público. Bens e serviços assim oferecidos à população maringaense também devem estar influindo favoravelmente na qualidade de vida. Além disso, Maringá não conta com grandes conglomcrados populacionais $\mathrm{em}$ precárias condições de vida favelas ou cortiços - tão comuns, hoje, nas cidades. A população rural, bastante sacrificada na conjuntura atual, é limitada a $2 \%$. Observa. sc, portanto, que as camadas sociais nas quais a mortalidade infantil é geralmente alta, em conseqüência do nívcl de vida, significam uma parcela reduzida da população.

\section{Probabilidade de morte infantil segundo subgrupos etários e causa básica}

Os óbitos que ocorreram em menores de um ano concentraram-se, principalmente, no periodo nconatal $(77,3 \%)$ (Tabela 1) e foram, em sua maioria, devido às causas perinatais (Tabela 2), com baixa frequiência de doenças infecciosas. Observa-se na Tabela 1 que, enquanto a probabilidade de morte infantil tardia foi de 4,5 por mil, a neonatal foi 15,4 .

Em $63,8 \%$ dos 6 bitos infantis a causa básica foi afecções originadas no período perinatal (Tabela 2) e a probabilidade de morte foi de 12,3 por mil. Victora e col. ${ }^{21}$, ao comentar a participação das causas perinatais na mortalidade infantil em Pelotas (1982), as comparam com os dados da Inglaterra e Pais de Gales, onde, a exemplo de Pelotas, as patologias perinatais foram as principais causas de morte. No entanto, o coeficiente em Pelotas foi 3,7 vezes maior (16,6 por mil n.v. c 3,5 por mil n.v.,

Tabela 1. Número e percentagem de óbitos e probabilidade* de morte de menores de 1 ano (por mil) segundo subgrupos etários. Maringá-PR, 1989.

\begin{tabular}{lccc}
\hline Subgrupos etários & \multicolumn{2}{c}{ Óbitos } & $\begin{array}{c}\text { Probabilidade* } \\
\text { de morte }\end{array}$ \\
\cline { 2 - 3 } & Ne & $\%$ & \\
\hline Neonatal precoce & 61 & 62,9 & 12,5 \\
Neonatal tardia & 14 & 14,4 & 2,9 \\
Pós-neonatal & 22 & 22,7 & 4,5 \\
\hline Total & 97 & 100,0 & 19,9 \\
\hline
\end{tabular}

- Calculadas em relação ao total de 4.876 nascidos vivos.

Tabela 2. Número e percentagem de óbitos de menores de 1 ano segundo grupos de causas* básicas $e$ subgrupos etários. Maringá-PR, 1989.

\begin{tabular}{|c|c|c|c|c|c|c|}
\hline \multirow[b]{3}{*}{ Grupos de causas** } & \multicolumn{4}{|c|}{ Subgrupos } & & \\
\hline & \multicolumn{2}{|c|}{ Neonatal } & \multicolumn{2}{|c|}{ Pós-neonatal } & \multicolumn{2}{|c|}{ Total } \\
\hline & $N^{2}$ & $\%$ & $N^{2}$ & $\%$ & $N^{2}$ & $\%$ \\
\hline $\begin{array}{l}\text { I. Doenças infecciosas e parasitárias } \\
\text { II. Neoplasmas } \\
\text { III. D. glând. endóc., nutr. metab. e transt. imunitários } \\
\text { VI. Doenças do sistema nervoso e órgãos dos sentidos } \\
\text { VIII. Doenças do aparelho respiratório } \\
\text { IX. Doenças do aparelho digestivo } \\
\text { XIX. Anomalias congênitas } \\
\text { XV. Algumas afecçōes originadas no periodo perinatal } \\
\text { XVI. Sintomas, sinais e afecçōes mal definidas } \\
\text { XVII. Causas externas }\end{array}$ & $\begin{array}{l}- \\
- \\
- \\
- \\
- \\
11 \\
60 \\
1 \\
-\end{array}$ & $\begin{array}{l}- \\
- \\
- \\
- \\
- \\
15,3 \\
83,3 \\
1,4 \\
-\end{array}$ & $\begin{array}{l}1 \\
2 \\
1 \\
1 \\
2 \\
1 \\
8 \\
- \\
2 \\
4\end{array}$ & $\begin{array}{r}4,5 \\
9,1 \\
4,5 \\
4,5 \\
9,1 \\
4,5 \\
36,4 \\
- \\
9,1 \\
18,1\end{array}$ & $\begin{array}{r}1 \\
2 \\
1 \\
1 \\
2 \\
1 \\
19 \\
60 \\
3 \\
4\end{array}$ & $\begin{array}{r}1,1 \\
2,1 \\
1,1 \\
1,1 \\
2,1 \\
1,1 \\
20,2 \\
63,8 \\
3,2 \\
4,3\end{array}$ \\
\hline Total & 72 & 100,0 & 22 & 99,8 & $94^{* * *}$ & 100,1 \\
\hline
\end{tabular}

- Segundo os capítulos da $\mathrm{CID}^{13}, 9^{9}$ revisão.

** Somente capítulos cuja freqüência é diferente de zero.

** Excluídos 3 casos cujas causas básicas sãoignoradas. 
respectivamente). Para o ano de 1989, no Estado do Rio Grande do Sul, 47,6\% dos óbitos de menores de 1 ano livcram como causa básica essas afecções ${ }^{16}$; em Santos, $61,7 \%$ dos falecidos aprescntaram esta afecção*. Em Maringá, todos os óbitos por esta causa ocorreram até o $28^{\circ}$ dia de vida $\mathrm{c}$ responderam por mais de $80 \%$ dos óbitos do período nconatal. Na Tabela 3 é possível verificar que $20 \%$ dos óbitos relativos às afecções originadas no período perinatal apresentaram como causa básica o crescimento fetal retardado, a má nutrição c a prematuridade e $48,3 \%$, a hipóxia, anóxia ao nascer ou outras afecçð̃es respiratórias.

Tabela 3. Distribuição do número e percentagem de óbitos neonatais segundo tipo de afocçōes originárias no periodo perinatal*. Maringá-PR, 1989.

\begin{tabular}{lrr}
\hline Tipo de afecçōes & N & $\%$ \\
\hline Crescimento fetal retardado, mal nutrição & & \\
fetal e prematuridade (764-765) & 12 & 20,0 \\
Trauma de parto (767) & 2 & 3,3 \\
Hipóxia, anóxia ao nascer e outras & 29 & 48,3 \\
afecçōes respiratórias (768-770) & 29 \\
Doença hemolítica do teto ou do recém- & 1 & 1,7 \\
nascido & 16 & 26,7 \\
Outras & 60 & 100,0 \\
\hline Total & &
\end{tabular}

- Rubricas de três algarismos da Lista Brasileira para Mortalidade, correspondente ao capítulo XII da CID-9 (categorias 760-779).

Essas causas de óbilos sugerem que uma significativa parcela de produtos de concepção ainda prematuros vicram a nascer. Excmplo disso é a menção da imaturidade ou prematuridade, como causa básica** ou associada, cm $38,1 \%$ das DO cstudadas, percentagem que passa para $49,2 \%$ quando se consideram as do período nconatal, ou ainda, $68,3 \%$ daquclas devido às causas perinatais. Rcforça ainda a idćia de imaturidade/prematuridade, a alta freqüência de nascimentos de baixo peso cntre as crianças falccidas por essas causas; ou scja, $71,7 \%$ tinham peso inferior a $2.500 \mathrm{~g}$, proporção que passa para $81,7 \%$ quando se consideram tambím as de peso inadequado.

Estas questões levam a questionar a qualidade clos scrviços de saúde voltados para assistência pré-natal, ao parto e ao recém-nascido. Em alguns casos a imaturidade $\mathrm{c}$ nascimentos de baixo peso são conscqüicntes a problemas evitáveis através de intervençöcs adequadas. Ressalte-se a influência das cesarianas na mortalidade obscrvada, pois a retirada da criança $\mathrm{cm}$ uma fase precoce, ainda vulncrávcl fisiologicamente, pode representar risco à criança. As probabilidades de morte em menores de um ano por afecções originárias no período perinatal, segundo tipo de parto, foram, respectivamente, 20,3 (parto normal) e 9,0 (parto cesariano) por mil. No entanto, cabe a ressalva de que a maior proporção de partos normais em crianças de menores pesos (Tabela 4) pode também ser indicativa dc negligência no atendimento e pior nível socioeconômico. Em muitos casos, um controle c acompanhamento adequado das gestações de alto risco poderiam prevenir nascimentos de baixo peso.

Tabela 4. Número e percentagem de nascidos vivos em hospitais, filhos de mulheres residentes, segundo peso ao nascer (em g) e tipo de parto. Maringá-PR, 1989.

\begin{tabular}{|c|c|c|c|c|c|c|}
\hline \multirow{3}{*}{$\begin{array}{l}\text { Peso ao } \\
\text { nascer }\end{array}$} & \multicolumn{4}{|c|}{ Parto } & \multirow{2}{*}{\multicolumn{2}{|c|}{ Total }} \\
\hline & \multicolumn{2}{|c|}{ Normal } & \multicolumn{2}{|c|}{ Cesárea } & & \\
\hline & $N^{8}$ & $\%$ & $N^{2}$ & $\%$ & $N^{2}$ & $\%$ \\
\hline $\begin{array}{r}400 \text { a } 1.499 \\
1.500 \text { a } 1.999 \\
2.000 \text { a } 2.499 \\
2.500 \text { a } 2.999 \\
3.000 \text { a } 3.499 \\
3.500 \text { a } 3.999 \\
4.000 \text { a } 4.900\end{array}$ & $\begin{array}{r}21 \\
25 \\
82 \\
406 \\
578 \\
277 \\
40\end{array}$ & $\begin{array}{l}63,6 \\
46,3 \\
32,4 \\
35,7 \\
28,0 \\
24,8 \\
19,6\end{array}$ & $\begin{array}{r}12 \\
29 \\
171 \\
732 \\
1.488 \\
840 \\
164\end{array}$ & $\begin{array}{l}36,4 \\
53,7 \\
67,6 \\
64,3 \\
72,0 \\
75,2 \\
80,4\end{array}$ & $\begin{array}{r}33 \\
54 \\
253 \\
1.138 \\
2.066 \\
1.117 \\
204\end{array}$ & $\begin{array}{l}100,0 \\
100,0 \\
100,0 \\
100,0 \\
100,0 \\
100,0 \\
100,0\end{array}$ \\
\hline Tolal & 1.429 & 29,4 & 3.439 & 70,6 & 4.865 & 100,0 \\
\hline
\end{tabular}

* Excluídos 11 casos cujo tipo de parto elou peso ao nascer são ignorados.

Estas questões sugerem possibilidade de diminuição de óbitos por causas perinatais. A cxtensão de cobcrtura dos serviços voltados para o grupo matcrno-infantil (como ocorrcu $\mathrm{cm}$ Maringá $\mathrm{e} \mathrm{cm}$ várias regiõcs brasilciras, scja atravćs de programas oficiais como PAISMC***, ou iniciativas isoladas inscridas $\mathrm{cm}$ planos municipais de saúde), ocasionou pouco impacto na mortalidade neonatal, icndo scu maior reflexo sobre a mortalidade pós-nconatil.

Obscrva-sc que, cm Maringá cm 1989, as anomalias congênitas foram responsávcis por $36,4 \%$ dos óbitos ocorridos no período pós-nconatal (Tabela 2) c as docnças infecciosas e respiratórias,

\footnotetext{
* Trabalho apresentado no I Congresso Brasilciro de Epidemiologia, Campinas, 1990, na Scssão de "Comunicaçôcs Coordenadas".

** Scgundo regras da Classificação Intemacional de Doenças, a prematuridade só ć considcrada causa básica quando não há menção a outra patologia na declaração de óbito.

*** Programa de Assistência Integral à Saúde da Mulher e da Criança, implantado no país sob a coordenação do Ministćrio da Saúde, na década de 80.
} 
bastante importantes em anos antcriores, foram mencionadas como causas básicas em apenas $4,5 \%$ c $9,1 \%$, respectivamente, no pcríodo. Essas percentagens passam para 1,1 e 2,1 , quando se considcram os óbitos do primciro ano de vida. Além disso, outras causas que ocupavam posiçõcs menos importantes, como as causas extcrnas, começam a merecer atenção, sendo responsávcis por $4,3 \%$ dos óbitos infantis (um devido a acidente de trânsito c três por asfixia mecânica).

Assiste-sc atualmente a mudanças nas causas de óbito; as docnças infecciosas e respiratórias, até então, relevantes no quadro de mortalidade infantil, passam a scr gradativamente substituídas pelas não-infecciosas, fato que tem sido denominado de transição epidemiológica ${ }^{10}$.

\section{Probabilidade de morte infantil segundo sexo}

Paradoxalmente aos resultados citados $\mathrm{em}$ vários estudos (onde há sempre sobremortalidade infantil masculina), entrc as crianças nascidas, $\mathrm{cm} 1989, \mathrm{~cm}$ hospitais de Maringá, as do sexo feminino apresentaram risco de morrer 1,4 vezes o correspondente ao do scxo masculino (Tabela 5). Este diferencial no período nconatal foi de $33,3 \%$, c a partir do $29^{\circ}$ dia de vida foi de $72,7 \%$ maior no sexo feminino.

Tabela 5. Óbitos e probabilidade de morte de menores de 1 ano (por mil) segundo sexo e subgrupos etários. Maringá-PR, 1989.

\begin{tabular}{|c|c|c|c|c|c|c|}
\hline \multirow[t]{2}{*}{ Sexo } & \multicolumn{2}{|c|}{ Neonatal } & \multicolumn{2}{|c|}{ Pós-neonatal } & \multicolumn{2}{|c|}{ Total } \\
\hline & $N^{2}$ & Prob & $N^{2}$ & Prob & $N^{Q}$ & Prob \\
\hline $\begin{array}{l}\text { Masculino (2.431) } \\
\text { Feminino (2.438) }\end{array}$ & $\begin{array}{l}32 \\
43\end{array}$ & $\begin{array}{l}13,2 \\
17,6\end{array}$ & $\begin{array}{r}8 \\
14\end{array}$ & $\begin{array}{l}3,3 \\
5,7\end{array}$ & $\begin{array}{l}40 \\
57\end{array}$ & $\begin{array}{l}16,5 \\
23,4\end{array}$ \\
\hline Total (4.869) & 75 & 15,4 & 22 & 4,5 & 97 & 19,9 \\
\hline
\end{tabular}

Entre parênteses, totais de nascidos vivos segundo sexo.
Fcrrcira ${ }^{5}$, analisando a influência do sexo na mortalidade infantil, ressalta maior vulnerabilidade masculina e mostra que no período de 1979/ 1984 a MI masculina no Estado de São Paulo foi supcrior à feminina em $30 \%$, diferença esta maior no período nconatal (1,36 vezes). Baseado em alguns autores, explica a sobremortalidade masculina a partir do "processo de maturação dos pulmōes das crianças do sexo masculino que é mais lento durante o descnvolvimento fetal (... )" c que o "diferencial por sexo da mortalidade nconatal é, em geral, maior do que o da pós-neonatal (...). Por outro lado, a ocorrência de una maior mortalidade infantil feminina está mais associada a questöes socioculturais de discriminação ao sexo feminino, que resultam em prejllizos aos cuidados e atenção às crianças deste sexo...". Este último fator, provavelmente, não deveria estar influenciando maior mortalidade infantil feminina obscrvada, pois não há cvidências para tal fato; o resultado encontrado para Maringá recomenda cautcla quanto às conclusões. A sobrcmortalidade feminina observada deve ser apenas achado casual para o ano de 1989. À guisa de csclarecimento, cm sćric histórica de 1980 a 1989, referente à Maringá, pode ser obscrvado que cm 1982 tal fenômeno também ocorreu, sendo que, dos óbilos menores de um ano, 70 óbitos cram do sexo masculino e 74 referiam ao sexo feminino.

\section{Probabilidade de morte infantil segundo peso ao nascer}

O baixo peso ao nascer, devido a sua influência na sobrevivência infantil, $\mathrm{tcm}$ sido objcto de várias investigaçõcs. Verifica-sc que, entre os nascimentos hospitalares ocorridos cm Maringá, $\mathrm{cm} 1989$, à medida que aumenta o peso diminui a probabilidade de morrer (Tabela 6). Enquanto as crianças nascidas com peso inferior a $1.500 \mathrm{~g}$ apresentam probabilidade de morte no primciro

Tabela 6. $N^{0}$ de nascidos vivos, $n^{2}$ de óbitos menores de 1 ano e probabilidade de morte de menores de um ano de vida (por mil) segundo sexo e poso ao nascer (em g). Maringá-PR, 1989.

\begin{tabular}{|c|c|c|c|c|c|c|c|c|c|}
\hline \multirow{3}{*}{ Peso ao nascer } & \multicolumn{6}{|c|}{ Sexo } & & & \\
\hline & \multicolumn{3}{|c|}{ Masculino } & \multicolumn{3}{|c|}{ Feminino } & \multicolumn{3}{|c|}{ Tolal } \\
\hline & $N^{Q}$ n.v & $N^{Q}$ ób. & Prob. & $N^{\Omega}$ n.v & Neób. & Prob. & $N^{0} n \cdot v$ & $N^{2} 6 b$. & Prob. \\
\hline $\begin{array}{r}400 \text { a } 1.499 \\
1.500 \text { a } 1.999 \\
2.000 \text { a } 2.499 \\
2.500 \text { a } 2.999 \\
3.000 \text { a } 4.999\end{array}$ & $\begin{array}{r}14 \\
21 \\
110 \\
466 \\
1.814\end{array}$ & $\begin{array}{r}12 \\
8 \\
4 \\
6 \\
10\end{array}$ & $\begin{array}{r}857,1 \\
381,0 \\
36,4 \\
12,9 \\
5,5\end{array}$ & $\begin{array}{r}19 \\
33 \\
140 \\
672 \\
1.571\end{array}$ & $\begin{array}{r}13 \\
9 \\
7 \\
10 \\
18\end{array}$ & $\begin{array}{r}684,2 \\
272,7 \\
50,0 \\
14,9 \\
11,5\end{array}$ & $\begin{array}{r}33 \\
54 \\
250 \\
1.138 \\
3.385\end{array}$ & $\begin{array}{l}25 \\
17 \\
11 \\
16 \\
28\end{array}$ & $\begin{array}{r}757,6 \\
314,8 \\
44,0 \\
14,1 \\
8,3\end{array}$ \\
\hline Total & 2.425 & 40 & 16,5 & 2.435 & 57 & 23,4 & $4.860^{\circ}$ & 97 & 19,9 \\
\hline
\end{tabular}

* Excluidos 16 casos cujo peso ao nascer e/ou sexo são ignorados. 
Tabela 7. Ne de nascidos vivos, $n^{2}$ de óbitos menores de 28 dias e probabilidade de morte de menores de 28 dias de vida (por mil) segundo sexo e peso ao nascer (em g). Maringá-PR, 1989.

\begin{tabular}{|c|c|c|c|c|c|c|c|c|c|}
\hline \multirow{3}{*}{ Peso ao nascer } & \multicolumn{6}{|c|}{ Sexo } & & & \\
\hline & \multicolumn{3}{|c|}{ Masculino } & \multicolumn{3}{|c|}{ Feminino } & \multicolumn{3}{|c|}{ Total } \\
\hline & $N^{2} n \cdot v$ & $\mathrm{~N}^{2}$ ób. & Prob. & $N^{2}$ n.v & $N^{2}$ ób. & Prob. & $N^{2}$ n.v & Ne ób. & Prob. \\
\hline $\begin{array}{r}400 \text { a } 1.499 \\
1.500 \text { a } 1.999 \\
2.000 \text { a } 2.499 \\
2.500 \text { a } 2.999 \\
3.000 \text { a } 4.999\end{array}$ & $\begin{array}{r}14 \\
21 \\
110 \\
466 \\
1.814\end{array}$ & $\begin{array}{r}12 \\
7 \\
3 \\
4 \\
6\end{array}$ & $\begin{array}{r}857,1 \\
333,3 \\
27,3 \\
8,6 \\
3,3\end{array}$ & $\begin{array}{r}19 \\
33 \\
140 \\
672 \\
1.571\end{array}$ & $\begin{array}{r}13 \\
9 \\
7 \\
5 \\
9\end{array}$ & $\begin{array}{r}684,2 \\
272,7 \\
50,0 \\
7,4 \\
5,7\end{array}$ & $\begin{array}{r}33 \\
54 \\
250 \\
1.138 \\
3.385\end{array}$ & $\begin{array}{r}25 \\
16 \\
10 \\
9 \\
15\end{array}$ & $\begin{array}{r}757,6 \\
296,3 \\
40,0 \\
7,9 \\
4,4\end{array}$ \\
\hline Tolal & 2.425 & 32 & 13,2 & 2.435 & 43 & 17,6 & $4.860^{*}$ & 75 & 15,4 \\
\hline
\end{tabular}

- Excluídos 16 casos cujo peso ao nascer e/ou sexo são ignorados.

ano de vida de 757,6 por mil, entre aquelas nascidas com peso mínimo de $3.000 \mathrm{~g}$, a probabilidade foi de 8,3 por mil. A probabilidade de morte em crianças de baixo peso ao nascer, menores de um ano, foi de 155,9 por mil contrapondo-se a 9,7 por mil daquelas que nasceram com peso mínimo de $2.500 \mathrm{~g}(\mathrm{RR}=16)$.

Comparando os dados das Tabelas 6 e 7 verifica-se que todos os óbitos de crianças nascidas com peso inferior a $1.500 \mathrm{~g}$ ocorrcram no período nconatal, período bastante crítico no qual a sobrevivência depende da capacidade do recém-nascido supcrar, principalmente, os problemas decorrentes da imaturidade pulmonar.

Bobadilla e col. ${ }^{2}$ colocam que o pcso ao nascer constitui um eficiente prognóstico de morte perinatal e destacam-no como fator mais importante a explicar o cfcito das variáveis sociais e biológicas na mortalidade pcrinatal.

A problemática da mortalidade por baixo peso foi mais intcnsa nas crianças do sexo masculino. A probabilidade de morte obtida através da estratificação segundo sexo $\mathrm{e}$ peso ao nascer (Tabelas $6 \mathrm{c}$ 7) mostra que, cm nascimentos cujo peso foi inferior a $2.000 \mathrm{~g}$, o sexo masculino foi mais vulncrávcl, com risco aumentado $\mathrm{cm} 25,3 \%$ para nascidos com pesos atć $1.500 \mathrm{~g}$ c $39,7 \%$ para aqueles de peso cntre 1.500 e $1.999 \mathrm{~g}$. A partir de $2.000 \mathrm{~g}$ inverte-se a relação: a probabilidade de morte passa a ser maior no scxo feminino, atingindo, a partir de $3.000 \mathrm{~g}$, risco $100 \%$ maior. Observa-se, portanto, que o peso ao nascer tcm papel preponderante na mortalidade obscrvada (das crianças que nasceram com peso inferior a $1.500 \mathrm{~g}$, somente $24,2 \%$ sobrcvivcram). Comparando os dados das Tabclas $6 \mathrm{c} 7$, $c$ possível constatar que, mesmo nascendo neste intervalo de peso (até $1.500 \mathrm{~g}$ ), os meninos que resistiram até o $28^{\circ}$ dia sobreviveram ao primciro ano de vida. Entre as meninas nascidas com pcso ate $2.500 \mathrm{~g}$, o mesmo fato ocorrcu. Em relação aos nascidos vivos de maior peso, observa- se que a mortalidade referente ao período pósnconatal aumenta sua participação à medida que os pesos assumem maiores valores.

\section{Probabilidade de morte segundo idade materna}

A idade da mãe na ocasião do nascimento da criança constitui um importante fator para a mortalidade infantil. Os resultados da Investigação Interamericana de Mortalidade na Infância ${ }^{15}$ revclavam maior mortalidade neonatal em filhos de mulheres com menos de 20 anos, e aqueles cujas mães tinham idade entre 20 a 24 anos eram os mais protegidos. Mostraram, também, que a taxa de mortalidade infantil aumentava após os 35 anos de idade.

Os resultados para Maringá corroboram os achados da citada Investigação ${ }^{15}$. Conforme se obscrva na Tabela 8, a probabilidade da criança falecer no primeiro ano de vida foi de 71,4 por mil, quando suas mães tinham menos de 15 anos, probabilidade que tendeu a ser bem menor $(25,4$ por

Tabela 8. $N^{2}$ de nascidos vivos, $n^{2}$ de óbitos e probabilidade de morte de menores de um ano de vida (por mil) segundo idade materna e subgrupos etários. Maringá-PR, 1989.

\begin{tabular}{|c|c|c|c|c|c|c|}
\hline \multirow[t]{2}{*}{ Idade da mãe } & \multicolumn{2}{|c|}{ Neonatal } & \multicolumn{2}{|c|}{ Pós-neonatal } & \multicolumn{2}{|c|}{ Total } \\
\hline & $N^{2} 6 b$ & Prob & $N^{Q}$ ób & Prob & $N^{2}$ ób & Prob \\
\hline 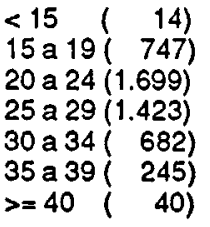 & $\begin{array}{r}1 \\
11 \\
25 \\
21 \\
11 \\
6 \\
-\end{array}$ & $\begin{array}{l}71,4 \\
14,7 \\
14,7 \\
14,8 \\
16,1 \\
24,5 \\
-\end{array}$ & $\begin{array}{l}- \\
8 \\
2 \\
8 \\
2 \\
1 \\
1\end{array}$ & $\begin{array}{r}- \\
10,7 \\
1,2 \\
5,6 \\
2,9 \\
4,1 \\
16,7\end{array}$ & $\begin{array}{r}1 \\
19 \\
27 \\
29 \\
13 \\
7 \\
1\end{array}$ & $\begin{array}{l}71,4 \\
25,4 \\
15,9 \\
20,4 \\
19,1 \\
28,6 \\
16,7\end{array}$ \\
\hline Total $\quad(4.870)$ & 75 & 15,4 & 22 & 4,5 & 97 & 19,9 \\
\hline
\end{tabular}

Entre parêntesis estão apresentados os números de nascidos vivos segundo idade da mäe, sendo que 6 casos foram excluídos pois a idade da mãe é ignorada. 
mil) na faixa imediatamente posterior (15 a 19 anos), atingindo melhores possibilidades de sobrcvivência entre 20 a 24 anos (risco de 15,9 por mil). O risco de morrer foi maior na faixa de 35 a 39 anos de idade (28,6 por mil).

Muitas explicaçoes são dadas a respeito da maior mortalidade nos nascimentos de mãcs $\mathrm{cm}$ idades extremas (adolescentes e aquelas com 35 anos e mais). Entre essas, são citados os problemas de natureza fisiológica, que se traduzem na maior ocorrência de patologias perinatais, nas maiores taxas de imaturidade e, conseqüentemente, no aumento da incidência de baixo peso ao nascer, que se observa com maior freqüência entre mãcs jovens ${ }^{4,5,15}$.

Ao distribuir os nascimentos segundo idade materna e peso ao nascer (Tabcla 9) mais uma vcz evidencia-se o esperado, isto é, a proporção de baixo peso foi superior nas idades extremas . Enquanto que $10,3 \%$ c $10,0 \%$ das mães com menos de 20 anos, e 40 anos ou mais, respectivamente, tiveram filhos com menos de $2.500 \mathrm{~g}$, somente $5,8 \%$ dos nascidos vivos, filhos de mulheres entre 20 c 25 anos de idade, apresentaram baixo peso.

Tabela 9. Distribuição do número e percentagem de nascidos vivos em hospitais, filhos de mulheres residentes, conforme a idade materna e peso ao nascer. Maringá-PR, 1989.

\begin{tabular}{|c|c|c|c|c|c|c|}
\hline \multirow{3}{*}{$\begin{array}{l}\text { Idade } \\
\text { malerna }\end{array}$} & \multicolumn{4}{|c|}{ Peso ao nascer } & & \\
\hline & \multicolumn{2}{|c|}{$<2.500 \mathrm{~g}$} & \multicolumn{2}{|c|}{$>=2.500 \mathrm{~g}$} & \multicolumn{2}{|c|}{ Tolal } \\
\hline & $\mathrm{N}^{2}$ & $\%$ & $\mathrm{~N}^{2}$ & $\%$ & $\mathrm{~N}^{2}$ & $\%$ \\
\hline $\begin{array}{l}<20 \\
20 \text { a } 24 \\
25 \text { a } 29 \\
30 \text { a } 34 \\
35 \text { a } 39 \\
>=40\end{array}$ & $\begin{array}{r}78 \\
99 \\
87 \\
49 \\
21 \\
6\end{array}$ & $\begin{array}{r}10,3 \\
5,8 \\
6,1 \\
7,2 \\
8,6 \\
10,0\end{array}$ & $\begin{array}{r}681 \\
1.595 \\
1.336 \\
629 \\
224 \\
54\end{array}$ & $\begin{array}{l}89,7 \\
94,2 \\
93,9 \\
92,8 \\
91,4 \\
90,0\end{array}$ & $\begin{array}{r}759 \\
1.694 \\
1.423 \\
678 \\
245 \\
60\end{array}$ & $\begin{array}{l}100,0 \\
100,0 \\
100,0 \\
100,0 \\
100,0 \\
100,0\end{array}$ \\
\hline Total & 340 & 7,0 & 4.519 & 93,0 & $4.859^{*}$ & 100,0 \\
\hline
\end{tabular}

- Excluídos 17 casos cuja idade materna e/ou peso ao nascer são ignorados.

Ao problema da maior mortalidade infantil, $\mathrm{cm}$ filhos de mulheres $\mathrm{cm}$ idades extremas, alem das razõcs já citadas, associa-se a de natureza sociocullural. Entre mãcs $\mathrm{cm}$ idades avançadas são mais freqüentes as grandes multiparas cujos intervalos interpartais inadequados podem diminuir as possibilidades de sobrevivência das crianças. A exemplo das adolescentes, cstas advĉm principalmente de camadas sociocconômicas mais desfavorccidas $\mathrm{cm}$ que, aos aspectos negativos nutricionais acumulam-sc menor acesso às informaçōes (scja $\mathrm{cm}$ nivel de sistema formal ou informal) e a ausência de outros fatores que contribuiriam favoravelmente na saúde infantil.

\section{Consideraçőes finais}

A mortalidade infantil continua sendo de grande importância no contexto atual e possivelmente constituir-se-á em objeto de vários estudos futuros, tendo em vista os seus múltiplos determinantes e a situação sociopolitica contemporânea. $O$ presente estudo pretendeu estimar a magnitude desta mortalidade no Município de Maringá; no entanto, na busca de explicaçōes para os resultados encontrados, novos problemas e várias hipóteses foram levantadas. Algumas questöes, embora não conclusivas, devido à sua relcvância, merccem ser destacadas.

Assim, cm termos gerais, a análise dos diferenciais de mortalidade infantil e da tendência declinante, seja em nivel de estratos sociais, regiðes ou países, deve contemplar, além das questões tradicionalmente abordadas (como o desenvolvimento socioeconômico), as transições demográfica e epidemiológica. A transição que ora sc observa tem se caractcrizado pelas alterações nos padrões rcprodutivos e nos padróes de mortalidade (diminuição da mortalidade infantil e alterações nas causas de óbito) ${ }^{10,14}$.

Há de ser ressaltado o fato de a probabilidade de morrer em menores de um ano nascidos de parto normal ter sido maior do que a dos nascidos por parto tipo cesária. Entretanto, em trabalho antcrior $^{20}$, nesta população de nascidos vivos foi detectada associação entre ter nascido por parto normal e baixo nível socioeconômico da mãe; portanto, não é o tipo de parto que leva a um maior risco de morrer mas sim o nível socioeconômico e o atendimento.

Outro ponto a comentar é o quanto a organização administrativa e a expansão dos scrviços básicos de saúde, ocorridas na década de 80 , contribuíram para reduzir a mortalidade infantil aos níveis atuais em Maringá. Conforme revelaram os rcsultados, a maioria dos óbitos infantis, em 1989, ocorreram no período neonatal e tiveram como principal causa de óbito, as causas perinatais. Quando se analisou a evolução da mortalidade infantil, no período $1980 / 88$, verificou-se que a mortalidade neonatal apresentou redução pouco significaliva durante o periodo, apesar do aumento substancial da of crta de serviços, com atenção cspecial ao grupo materno-infantil (o que pressupōe uma assistência sistematizada às gestantes). Estas evidências sugerem a necessidade de avaliar a eficácia e a cficiência da assistência prestada, prin- 
cipalmente se se considcrar que reduções futuras na mortalidade infantil dependem da capacidade de resolver, inclusive, os problemas relativos às causas perinatais.

SOUZA, R.K.T. de \& GOTLIEB, S.L.D [Probability of dying in the first year of life in an urban area of Brazil]. Rev. Saúde Publica, 27: 445-54, 1993. A birth-cohort of 4,876 children born alive in hospital were selected and followed through up to the age of one year with a view to estimating the risk of dying in the first year of life. All of them were bom in 1989, in one of the the seven hospitals of an urban area of Southem Brazil and the only requirement for belonging to the cohort was that of residence on the area. The selected variables were: sex, birthweight, age at moment of death, urderlying cause of death, and matemal age. The estimated probability of dying in the first year was of 19.9 per 1,000 (77.3\% of the deaths occurred during the neonatal period). Perinatal causes and congenital malformations contributed to $80 \%$ of the deaths, and infectious diseases were the underlying cause of death in only $1.1 \%$ of the losses. The risk of dying in the first year of life due to afections arising during the perinatal period was higher among vaginally delivered babies $(20.3$ per 1,000$)$ than it was for those born by cacsarian section $(9$ per 1,000$)$. A higher probability of death was present among infants born to adolescent mothers, and those with low birthweight (less than $2,500 \mathrm{~g}$ ). The results brought out the need for improving the quality of prenatal and infant care. They also suggested the hypothesis of a possible association between higher infant mortality and lower socio-economic level.

Keywords: Infant mortality. Probability. Risk factors.

\section{Referênclas Bibliográficas}

1. AMIGO, II. et al. Mortalidade em menores de cinco anos na cidade de Recife, PE (Brasil): tendências e associaçōes. Rev. Sauide Publica, 19: 531-42, 1985.

2. BOBADILLA, J.L. et al. Los efectos de la calidad de la atención medica en la sobrevivencia perinatal. Salud Publi. ca Mex., 30: 416-31, 1988.

3. BREILII, J. ct al. Ciudad e muerte infantil. Quito, C.E.A.S., 1983.

4. BUCIIALLA, C.M. Estudo de um grupo de recóm-nascidos em matemidades: suas características e mortalidade no período nco-natal precoce. São Paulo, 1988. [Disscrtação deMestrado - Faculdade de Saúde Pública da USP].

5. FERREIRA, C.E.C. Mortalidade infantil e desigualdade social em São Paulo. São Paulo, 1990. [Tese de Doutora. do - Faculdade de Saúde Pública da USP].
6. HOGUE, C.J.R. et al. Overview of the national infant mortality surveillance (NTMS): project-design, methods, results. Public Health Rep., 102: 126-38, 1987.

7. KOWARICK, L. et al. Novas formas de pobreza e espaço urbano em São Paulo: a diminuição perversa da segregação socioespacial. In: Congresso Brasileiro de Epidemiologia, 12, Campinas, 1990. Anais. Rio de Janeiro, ABRASCO, 1990. p. 274-81.

8. LAURENTI, R. Mortalidade infantil nos Estados Unidos, Suécia e Estado de São Paulo. Rev. Saúde Pública, 21: 268-73, 1987.

9. LAURENTI, R. et al. Estatísticas de satide. São Paulo, E.P.U., 1987.

10. LAURENTI, R. Transição demográfica e transição epidemiológica. In: Congresso Brasileiro de Epidemiologia, 12. Campinas, 1990. Anais. Rio de Janeiro, ABRASCO, 1990. p. 143-63.

11. MONTEIRO, C.A. Mortalidade infantil e desenvolvimento social. Saúde Deb., 5 (10): 27-9, 1980.

12. MONTEIRO, C.A. Contribuição para o estudo do significado da evolução do coeficiente de mortalidade infantil no Município de Sāo Paulo, SP (Brasil), nas três últimas décadas (1950-1979). Rev. Saúde Pública, 16: 7-18, 1982

13. ORGANIZAÇĀO MUNDIAL DA SAÚDE. Manual da classificação estatística internacional de doenças, lesöes e causas de óbilo; 9 rev. 1975. São Paulo, Centro da OMS para Classificação de Doenças em Português, 1879. v. 1 .

14. PATARRA, N.L. Transição em marcha: novas questōes demográficas. In: Congresso Brasileiro de Epidemiologia, 19. Campinas, 1990. Anais. Rio de Janeiro, ABRASCO, 1990. p. 187-97.

15. PUFFER, R.R. \& SERRANO, C.V. Características de la mortalidad en la niñez. Washington, Organizacion Panamericana de la Salud, 1973.

16. SECRETARIA DE SAÚDE E MEIO AMBIENTE. A criança no Rio Grande do Sul: indicadores de saude. Porto Alegre, 1990.

17. SÁ, X. Qualidade de vida mostra dois "Brasis" em São Pau. lo. Folha de Säo Paulo, S.Paulo, 05 jan. 1992, p. 8.

18. SIMŌES, C.C. da \& OLIVEIRA, L.A.P. de Evolução da mortalidade infantil. In: Fundação IBGE. Perfil estalístico de crianças e mäes no Brasil: aspectos socioeconômicos da morlalidade infantil em areas urbanas. Rio de Janeiro,1986. p. 29-48.

19. SIMÖES, C.C., org. Perfil estatístico de crianças e mäes no Brasil: mortalidade infantil e saúde na década de 80. Rio de Janeiro, IBGE, 1989.

20. SOUZA, R.K.T. de \& GOTLIEB, S.L.D. Sub-registro de nascimentos vivos hospitalares em área urbana da rcgião sul do Brasil, em 1989. Rev.Saúde Pública, 27: 177-84, 1993.

21. VICTORA, C.G. et al. Epidemiologia da desigualdade. $2^{a}$ ed. São Paulo, IIUCITEC, 1989.

Recebido para publicaçäo em 20,4.1993 Reapresentado em 25.8.1993

Aprovado para publicaçäo em 20.9.1993 